\title{
The mitotic cycle and cell population control
}

\author{
L. F. LAMERTON
}

From the Biophysics Division, Institute of Cancer Research, Sutton, Surrey

There is a danger, in the study of the mechanisms of control of mitosis, of oversimplifying the problems. This is particularly so in attempts to extract controlling substances from tissues where the procedures used may take no account of the structural heterogeneity of the system. It has to be recognized that in many tissues one will be dealing with a number of subpopulations, related to each other in precursor sequence or in tunction, but of different proliferative characteristics and potential and responding to different types of stimulus.

It is therefore important that a study of the structure and proliferative characteristics of cell populations in vivo should run parallel with fundamental biochemical studies if a proper understanding of proliferation control is to be achieved. This paper is intended as a very brief survey of what might be called the dynamic histology of tissues with some indication of its implications for mechanisms of control of mitosis.

\section{Proliferative Patterns in Normal Tissues}

There is a great variation in the rate of proliferation of cells in different tissues of the body. In normal, unstimulated tissues of rat or mouse the mean intermitotic time varies from hundreds of days in conditional renewal tissues such as the liver to 10 hours or so in certain cell populations, such as those found in the small intestine or among the later maturing blood cell precursors in the bone marrow. Some generalizations can, however, be made, particularly concerning the rate of division of the 'stem cells', that is, the members of the progenitor compartments which have the capacity both for selfmaintenance and for providing cells for the maturation pathways. It would appear, on the basis of tissues so far studied, that the stem cells, in the unstimulated state, are among the more slowly dividing cell populations found in tissues or, at least, contain a slowly dividing subpopulation. For instance, in the skin, the turnover time in the basal layer, which is composed mainly of stem cells, is of the order of several days in the rodent and considerably longer in man. In the bone marrow, where the stem cells cannot yet be distinguished morpho- logically, but can be studied by functional tests, the turnover time in the rodent is several days or more. 'Turnover time' is defined here as the time required for the cell population to produce a number of cells equal to the number already present. It is a measure of rate of cell production, and does not necessarily indicate the mean intermitotic time of the dividing cells, since it is a function of the proportion of the population which is dividing, as well as of the intermitotic time of the dividing cells.

In the small intestine, a tissue in which the cell kinetics have been studied extensively (Fry, Lesher, Kisieleski, and Sacher, 1963; Cairnie, 1967), it is not yet clear whether the stem-cell compartment comprises all cells in the proliferative zone of the crypts of Lieberkuhn or only those at the bottom. In the crypt of the small intestine it has recently been shown (Wright, Morley, and Appleton, 1972 and personal communication), both for the rodent and for man, that although most of the cells in the proliferating zone are dividing rapidly, those at the bottom have a much longer turnover time. Similarly, in the cartilage plate of the young rat, it has been shown that the cells at the top of the column are turning over much more slowly than those lower down (Kember, 1960). Thus in these tissues the stem-cell compartment, if not dividing slowly as a whole, at least contains a slowly dividing component.

Perhaps the clearest example of a stem-cell population of long turnover time is found in the conditional renewal tissues-such as the liver, kidney, thyroid, and salivary gland-where the turnover time is normally of the order of hundreds of days, though it is reduced dramatically under the appropriate stimulus.

When cells leave the stem-cell compartment and enter the maturation pathway they either cease division or continue to divide for a limited time, depending on the particular tissue. In the case of the skin the probability of division is greatly reduced, possibly to zero, when a cell leaves the basal layer. On the other hand, the bone marrow is a tissue in which many cell divisions intervene between the stem cell and the mature cell. It is interesting that the rate of cell division in the later precursors of the blood cells is, in unstimulated conditions, much 
more rapid than that of the stem cells. For example, in rats, the recognizable red cell precursors from the pronormoblast to the polychromatic stage divide with an intermitotic time between 10 and 12 hours (Hanna, Tarbutt, and Lamerton, 1969). Cessation of cell division, which occurs in the polychromatic stage, seems to occur without any substantial lengthening of the intermitotic time.

If the stem-cell compartment of the small intestine is confined to the base of the crypts, the remaining cells of the proliferation zone will represent a maturation compartment in which again the rate of cell division is much higher than that of the stem cells. Also, as with the bone marrow, there appears to be a rapid 'switch off' of cell division without lengthening of the cell cycle. Any broad generalizations about the proliferation characteristics of maturing cells must, however, await data on other tissues.

\section{Kinetic State of Unstimulated Stem Cells}

A relatively long turnover time, such as is shown by the stem-cell compartments of unstimulated tissues, could be explained in a number of ways. One possibility is that only a small fraction of the cells have division potential, though experimental data would argue against this. Alternatively, the cells may have to wait, on the average, a relatively long time after mitosis (in the so-called $\mathrm{G}_{0}$ state) before being triggered into preparation for division, or they may be progressing slowly through some stage of the cell cycle, or temporarily arrested. With available techniques it is often difficult, and sometimes not possible, to distinguish between these various alternatives, and particularly to distinguish between the possibilities in the pre-synthetic period, that is, $G_{0}$, slow progression through $G_{1}$, or arrest in $G_{1}$, though such information will be vital to the understanding of proliferation control if different control mechanisms are required to operate at different stages of the cell cycle.

In the liver and other conditional renewal tissues the evidence points to most of the cells being a true $\mathrm{G}_{0}$ state, awaiting the trigger to start preparation for DNA synthesis, but in the renewal tissues such as skin and bone marrow there is much doubt as to the kinetic state of stem cells, in spite of statements frequently encountered in the literature that they exist largely in the $\mathrm{G}_{0}$ condition.

The speed with which labelled cells or mitotic figures appear after a stimulus will give some information on the kinetic state of the cells responding. With the conditional renewal tissues there is an interval of about 18 to 20 hours between the stimulus and the start of DNA synthesis (Baserga, 1968; Threfall, 1968). An explanation for this, assuming the cells to be normally in the untriggered $G_{0}$ state, is that the interval of 20 hours represents the time after stimulus needed to complete preparation for DNA synthesis. The reason why all cells do not have a $G_{1}$ phase of 20 hours could be, as Baserga has suggested, because the enzymes necessary for the process of DNA synthesis decay with time, and that the length of the preparation period depends on the interval between the end of the previous division and triggering into preparation for DNA synthesis. With regard to the stem cells of the normal renewal tissues of the body, work on the skin (Block, Seiter, and Oehlert, 1963) and on bone marrow (Gidali and Lajtha, 1972; Lahiri and van Putten, 1972) has indicated that a substantial increase in labelled cells and mitotic figures can, under certain conditions, occur within a very few hours of the stimulus. This suggests that the stem cells of the normal renewal tissues contain at least some cells in $G_{1}$ or $G_{2}$, or in both, that can quickly complete the stages to mitosis. Evidence that a variety of normal tissues contain cells arrested in the $G_{2}$ phase has been provided by Pederson and Gelfant (1970). However, the recent paper of Hegazy and Fowler (1973) indicates that a relatively long intermitotic time does not necessarily indicate the presence of 'resting' or 'arrest' stages. By the method of percentage labelled mitoses they demonstrated, in unstimulated mouse skin, a narrow distribution of intermitotic time of basal layer cells around a value of about 110 hours. How far the duration of continued progress around the cell cycle can be extended is not known.

\section{Changes in Proliferative Pattern of Normal Tissues under Stimulation}

An increase in the rate of cell production in tissues, under an appropriate stimulus, is characterized by one or both of two types of change: (1) a shortening of the intermitotic time of cells, and (2) an extension of the zone of proliferation. The relative importance of these two types of change varies with the tissue and, in certain cases, with the nature of the stimulation. The normal response of the skin is a speeding up of cell proliferation in the basal layer with minimal, if any, extension of the proliferative zone above this layer. In the small intestine, when recovering from radiation damage, the most marked effect is an extension of the zone of proliferation up the crypt, though there is also some shortening of the intermitotic time of the dividing cells. A similar extension of the zone of proliferation has been shown 
by Zajicek in recent work on the inner enamel epithelium of the rodent incisor tooth, but here there is also a substantial shortening of intermitotic time among the dividing cells (Zajicek, Michaeli, and Weinreb, 1972). In the bone marrow there have now been many demonstrations of the speeding up of the stem cells that can occur in regenerative conditions, reducing the intermitotic time in rats and mice to less than 12 hours, perhaps even to values as short as six hours. In the maturation stages of cells in the bone marrow, a substantial shortening of intermitotic time can also occur. In addition, the equivalent of an extension of the zone of proliferation has been demonstrated, under conditions of continuous irradiation, when the number of cell divisions is increased in the recognizable precursors of the red cell series (and also possibly of the granulocyte series), thus increasing the 'amplification factor' of the later stages of maturation, defined as the number of mature cells produced for each cell entering the particular compartment (Tarbutt, 1969).

The bone marrow also provides a good example of the principle that, in the same tissue, different types of perturbation may call into play different types of homeostatic mechanism. Two quite distinct types of perturbation can be applied to this tissue. By radiation, or by the administration of certain classes of cytotoxic drugs, it is possible to damage specifically the proliferating precursors of the series, with little effect on the later non-dividing precursors, or on the mature cells. By bleeding, or by administration of phenylhydrazine in the case of red cells, it is possible to deplete the number of circulating mature cells, without depleting the precursor compartments.

The difference in response of the red cell system to these two types of perturbation is clearly demonstrated when the perturbations are continuously maintained by continuous irradiation in the one case, and by repeated administration of phenylhydrazine in the other (Tarbutt, 1969). Under these conditions new steady states are set up. In the rat it has been shown that the response to continuous irradiation is a speeding up of proliferation in the red cell stem-cell compartment, with an increase in transit time and number of divisions in the later, recognizable precursor compartment, allowing an increased amplification factor. Under maintained anaemia, however, the proliferation rate of the stem cells is not affected, at least for a time, and the amplification factor in the recognizable precursor compartment is unchanged. The increased rate of production of mature cells is the result of an increased flow of cells out of the erythropoietin sensitive cell compartment, which precedes the recognizable precursors, under the action of erythropoietin.

\section{Shortening of the Phases of the Cell Cycle}

When the intermitotic time is shortened during stimulation of a tissue, the pre-synthetic period generally shows the greatest absolute change. This is illustrated in table I.

\begin{tabular}{lllll}
\hline & $\begin{array}{l}\text { Intermitotic } \\
\text { Time in Hours }\end{array}$ & \multicolumn{2}{l}{ Duration of Phases in Hours } \\
\cline { 3 - 5 } & & $S$ & $G_{1}+\frac{1}{2} M$ & $G_{2}+\frac{1}{2} M$ \\
\hline Control mice & 42 & $8 \cdot 5$ & 32 & 1.5 \\
Stimulated mice & 26 & 5 & 19 & 1.5 \\
\hline
\end{tabular}

Table I The effect of oestrogen stimulation on the uterine epithelium of the castrated mouse (Epifanova, 1966)

The shortening of intermitotic time is, however, not confined to the pre-synthetic period. The duration of S may also show a considerable proportionate reduction, as in the example given above. Sometimes the absolute reduction in $\mathbf{S}$ may be large, as in the alveolar cells of the mammary gland in mice, where Bresciani (1965) has shown that the duration of $\mathbf{S}$ shortens from 21 hours to nine hours on stimulation with oestradiol-17 beta and progesterone.

Even in those rapidly dividing tissues where the duration of $G_{1}$ and $S$ are normally quite short, further reduction can occur with the appropriate stimulus. This is illustrated in table II.

\begin{tabular}{lllll}
\hline & $\begin{array}{l}\text { Intermitotic } \\
\text { Time in Hours }\end{array}$ & \multicolumn{2}{l}{ Duration of Phases in Hours } \\
\cline { 2 - 5 } & & $S$ & $G_{1}+\frac{1}{2} M$ & $G_{2}+\frac{1}{2} M$ \\
\hline Control rats & 9.9 & 6.7 & 1.6 & 0.7 \\
Anaemic rats & 6.9 & 5.5 & 0.4 & 0.9 \\
\hline
\end{tabular}

Table II The effect on the basophilic erythroblasts of the bone marrow of the rat kept anaemic by repeated injections of phenylhydrazine (Hanna et al, 1969)

\section{Control of Proliferation of Stem-cell Populations}

A basic question concerning stem-cell populations is how far their rate of proliferation is under the control of poietins or chalones or other agents originating from outside the compartment and how far it is determined by population-size control mechanisms from within the compartment. In view of the characteristic property of stem-cell populations to maintain a viable population size in the face of great variation in demand for cells for maturation, it is difficult, if not impossible, to devise a homeo- 
static mechanism which does not include some internal size control mechanism. Very little is known about how such mechanisms operate, but it is one field in which studies of the dynamic histology of tissues could make an important contribution.

An important experimental approach was initiated by Leblond and his colleagues (Marques-Pereira and Leblond, 1965; Leblond, Clermont, and Nadler, 1967) and followed up by Iversen and his colleagues (Iversen, Bjerknes, and Devik, 1968). They showed, by autoradiography of rat oesophageal epithelium or of mouse skin following tritiated thymidine, that when a cell in the basal layer divided the probability of one or both daughters moving out of the basal layer appeared to be largely a matter of chance. The observations could be interpreted in terms of cells being expelled from the basal layer as a result of population pressure. Iversen et al (1968) found that the probability of moving out appeared to be greater among the older cells than among those that had recently divided. Hegazy and Fowler (1973), however, found no evidence for such an age dependence; the probability of cells being squeezed out of the basal layer seemed to be purely random. These observations disproved the theory, at least for the skin, that homeostasis of the stem-cell population is achieved through a prior determination of the fate of the daughters of dividing stem cells, whether they should remain in the stem-cell compartment or move out onto the maturation pathway. The observations also suggest the possibility that a full stem-cell compartment may be necessary before cells can be released onto the maturation pathway, a concept that gains support from the fact that, when an area of skin is denuded of its epithelium by cauterization, a degree of covering of basal cells appears to be required before cell maturation starts, though other factors, such as fluid loss, could be playing a part here.

The concept that a full stem-cell compartment is necessary before cells are released for maturation is, in a number of respects, an attractive one. It makes the maintenance of stem-cell numbers a straightforward, self-limiting process, and the normal slow rate of division could be explained by the operation of some form of density-dependent inhibition of proliferation. It implies, of course, that there is a structure in the tissue that limits stem-cell activity to a given area or volume. The hypothesis has the disadvantage that response, in terms of mature cell output, must be delayed if the stem cells are depleted in numbers, though this may well be of less importance in the skin than in various other tissues.

Under this hypothesis the increase in proliferation rate that follows depletion of the later maturing or mature cells in the skin cannot be explained, as in the current chalone theory, by reduction in concentration of a proliferation inhibitor derived from the maturing cells. The feedback from the depleted mature or maturing cell compartment would have to be either a proliferative stimulus, which could overcome the effect of the density-dependent inhibition, or a stimulus for differentiation which could aid the release of cells from the basal layer. It is interesting that, in recent years, the 'chalone' theory of tissue population control has been based on control of proliferation but that a recent paper by Laurence, Randers-Hansen, Christophers, and Rytomaa (1972) revives the suggestion that 'chalones' may be primarily inhibitors of cell maturation.

It is not easy to see how, at the present time, one could test the hypothesis that a full stem-cell compartment in the skin is necessary before cells are released on the maturation pathway, and that the normal slow rate of proliferation in the stem-cell compartment of the skin is due to density-dependent factors. It will be a difficult theory to support if 'chalones' can be found in tissue which will inhibit the proliferation of depleted stem-cell populations in vivo. The fact that a tissue extract may reduce still further the proliferation rate of an already slowly dividing cell population does not constitute proof that it is responsible for the normal slow rate of proliferation.

One would also need to demonstrate that there is a structural containment of the stem-cell population. There are fundamental problems here concerning the relationship of cells in the basal layer with the basement membrane. Our ideas may have to be profoundly modified as a result of some recent observations (Mackenzie, 1970; Christophers, 1971) suggesting that the skin epithelium has a mosaic structure dividing it up into columns of cells, and that the distribution of mitoses in the basal layer and of cells moving out to maturation is related to the geometry of these columns.

Studies of proliferation in the stem-cell compartment of the bone marrow indicate that this differs from the skin in that it is much less dependent on changes in the populations of mature and maturing cells. In anaemia, as mentioned above, the main factor controlling the increase in red cell production appears to be the humoral agent erythropoietin, acting on the so-called 'erythropoietin-sensitive cells', which are a stage in development between the stem cells and the recognizable precursor cells. There is conflicting evidence, from work on rats and mice, as to whether it has any direct effect at all on the stem-cells. No change in proliferation rate of the stem-cells was demonstrated in rats with a maintained anaemia produced by repeated injections of phenyl- 
hydrazine (Blackett, 1968a). Nor does depletion of the recognizable red cell precursors in the bone marrow appear to affect the stem cells. My colleagues, Dr N. M. Blackett and Mr G. Standen, have shown that the rapidly dividing later precursors of red and white cell series in the rat bone marrow can be eliminated by daily doses of methotrexate and maintained in that state for at least 15 days without any measurable change in numbers or proliferation rate of the stem cells. On the other hand, it has frequently been demonstrated that the proliferation rate of the bone marrow stem cells is greatly increased following direct depletion of their numbers by radiation or cytotoxic drugs.

These observations point to an internal size control of proliferation rate of the stem cells and, as in the skin, one may consider the possibility of densitydependent mechanisms. However, very little is known about the microarchitecture of the bone marrow, which would provide a tissue structural basis for this hypothesis. The fact that in the bone marrow mature cells can be produced while the stem-cell population is greatly reduced suggests that, if release of cells from maturation requires a full stem-cell compartment, a 'multi-unit' system is necessary, similar to that found in the small intestine, where each unit (crypt of Lieberkuhn) is in essence a conservative system including both stem cells and maturing cells. Under such conditions, cells for maturation would be released as soon as individual units were filled up, although the total stem-cell population might be greatly depleted. There is little doubt that a knowledge of the details of the sinusoidal architecture and the spatial relationships between the precursors of the same and of different cell lines would help a great deal in understanding the mechanisms of cell proliferation and maturation in the bone marrow.

\section{Control of Proliferation of Maturing Cells}

In the bone marrow, as already mentioned, changes in proliferation kinetics can take place in the later stages of maturation of the red cells (and probably also of the white cells), altering the amplification factor in the maturation compartments. Under conditions of continuous irradiation, when there is a decreased flow rate into the recognizable precursor compartment, the transit time of cells within this compartment is lengthened and more divisions are inserted. Under maintained anaemia, when there is an increased flow into the compartment, the transit time is shortened, but the intermitotic time is also shortened, to yield an amplification factor that is not very different from normal (Blackett, 1968b). It might be possible to explain the changes in transit time by regarding mature cell production as essentially an overflow from a compartment which is spatially defined, but this is pure speculation, as is, at the present time, any explanation of the mechanisms for change in number of divisions or intermitotic time.

A change in number of divisions in the maturation sequence is evidence of a lack of close coupling between the proliferative and the maturation processes. Nevertheless, one has to ask how far, in the erythropoietic series, the content of the cell product, haemoglobin, affects the rate of cell proliferation. The lack of any substantial lengthening of the intermitotic time during the stages of maturation suggest that the effect is not considerable, though of course it is possible that there is a critical content of haemoglobin that causes cell division to cease abruptly.

The extension of the zone of cell proliferation following cell depletion is demonstrated very clearly in the crypt of the small intestine but investigation into the mechanisms, in terms of response of the various precursor compartments, is limited by the fact that the morphology of the cells shows so little change over the whole of the crypt. Galjaard, Meer-Fieggen, and Giesen (1972) have investigated the timing of this extension following irradiation. They have found evidence, with larger doses of radiation, that the time at which the extension starts is independent of the dose, and that it may be related to the transit time of cells along the villus, or at least, to some function of villus length.

\section{The Possible Role of Mechanical Pressure in vivo}

The possibility of deriving information about mechanisms of cell proliferation control from studies in vivo depends very much on finding ways by which the normal pattern of cell proliferation can be changed. The more specific the mode of action of the perturbing agent, the greater the chance of gaining useful information. In this context the work of Zajicek and his colleagues (Zajicek et al, 1972) on the inner enamel epithelium of the rat incisor tooth may well prove to be important in understanding the mechanism both of increase in rate of cell division and extension of the zone of proliferation. The inner enamel epithelium presents a cell arrangement somewhat similar to that of the small intestine, in that a clearly ordered arrangement of cells can be seen in histological sections, with the proliferating zone at one end, giving rise to a zone of nondividing cells and then to the material of the tooth itself. Zajicek found that the effect of shortening the incisor tooth, by cutting it, was to speed up the rate 


\begin{tabular}{|c|c|c|c|c|c|c|}
\hline & \multirow{2}{*}{$\begin{array}{l}\text { Proliferative } \\
\text { Compartment Size }\end{array}$} & \multirow{2}{*}{$\begin{array}{l}\text { Intermitotic } \\
\text { Time in Hours }\end{array}$} & \multicolumn{4}{|c|}{ Duration of Phases in Hours } \\
\hline & & & $S$ & $G_{1}$ & $M$ & $G_{8}$ \\
\hline $\begin{array}{l}\text { Control } \\
\text { Accelerated eruption }\end{array}$ & $\begin{array}{l}477 \pm 15 \text { cells } \\
608 \pm 20 \text { cells }\end{array}$ & $\begin{array}{l}23 \\
16\end{array}$ & $\begin{array}{l}7 \cdot 0 \\
5 \cdot 5\end{array}$ & $\begin{array}{r}14 \cdot 1 \\
8 \cdot 6\end{array}$ & $\begin{array}{l}0.4 \\
0.4\end{array}$ & $\begin{array}{l}1.5 \\
1.5\end{array}$ \\
\hline
\end{tabular}

Table III Effect of shortening incisor tooth on rate of cell division and on length of the proliferative zone

of division in the proliferative zone and to increase the length of the zone (table III).

It is difficult to see how cutting the tooth could affect the flow of some specific chemical messenger down into the proliferative zone. However, since the rat is continually grinding the incisor teeth of the upper and lower jaw, shortening of the tooth will reduce the mechanical pressure in the zones of cell proliferation and maturation. Thus here one may be dealing with a situation where mechanical pressure, presumably affecting the degree of contact between cells, could be an important factor in determining the proliferative state of the system.

There are other examples in vivo where rate of cell proliferation appears to be affected by cell density or mechanical pressure factors, for instance, in bone repair (Pritchard, 1963). The basic questions now are: (1) By what mechanisms do changes in cell density and cell contact exert an effect on the rate of cell proliferation? (2) How important are such effects compared with the operation of chemical messengers of various kinds?

The first of these questions is the subject of much experiment and theoretical work at the present time but whether the effects arise out of changes in cell motility or for other reasons is not yet established. With regard to the second question we cannot yet attempt an answer, and generalizations about the predominance of any one particular mechanism must be made with great caution.

\section{Factors Affecting Cell Proliferation in Tumours}

Tumour cell populations cannot be regarded as examples of 'uncontrolled' proliferation. In most primary tumours, in experimental animals, and in man the mean rate of cell division is low Irelative to that found in many normal renewal tissues of the body, and the spread in intermitotic time is generally considerable (Steel, 1972). It is reasonable to assume that deficiencies in oxygen and other nutrients due to inadequate vascularity, chromosomal abnormalities, and toxic products are important contributors to the slowing down of cell proliferation, although direct experimental evidence on this is sparse and somewhat conflicting. One would expect that many of the slowly dividing cells will be progressing towards sterility or death and therefore unimportant from the point of view of tumour growth or repopulation after damage. However, it is probable that some tumour 'stem cells'-cells that are capable of repopulating the tumour-are also to be found in the slowly dividing fraction, and are the reason for the poor response of some tumours to therapy using cycle-specific cytotoxic agents. From both a theoretical and practical point of view it is important to know the kinetic state of such cells. At the moment it is not possible to say whether they would be in an untriggered $G_{0}$ state or arrested or slowed down in the cell cycle, due possibly to nutritional deficiencies, though experimental work may soon provide some answers. It is conceivable that the type of tumour may have an influence: in the more highly differentiated tumours some tumour stem cells may retain some of the kinetic characteristics and susceptibility to control of the stem cells of the normal tissues, whereas in undifferentiated tumours nutritional deficiencies are likely to be a major factor.

In certain experimental and clinical observations (Hermens and Barendsen, 1969; Tubiana, 1971), a shortening of the intermitotic time of cells in tumours recovering from radiation-induced cell depletion has been demonstrated. This may be due entirely to adventitious factors resulting from products of cell death or changes in the vascularity of the tumour, but one cannot yet exclude the operation of other forms of control.

\section{Conclusions}

There are three main points emerging from this brief discussion of the dynamic histology of tissues which are important to the understanding of the control of cell proliferation in vivo.

The first is that the cell populations subject to control may exist in a variety of proliferative states, and the control mechanisms may differ, depending on whether a 'resting' cell has to be triggered into preparation for division, whether arrest at some stage within the cell cycle has to be overcome, whether cells already proliferating rapidly are to be speeded up, or whether the number of cell generations in a maturation sequence is to be increased. It 
would be very satisfying if one could associate these particular changes with particular types of feedback or density-dependent mechanisms of proliferation control, but the data are not yet available to attempt this, and there is still uncertainty about the normal proliferative state of the stem cells of the renewal tissues.

The second point is that the changes in stem-cell proliferation, observed in conditions of functional demand or damage, may, to an important extent, be the consequence of size control mechanisms within the cell compartment, operating as a result of cell depletion due to loss of cells for maturation or other cause. This has the implication that, in the search for feedback agents, the emphasis should perhaps be less on substances which directly affect stem-cell proliferation and more on agents directly initiating maturation and release from the stem-cell compartment.

The third point, related to the second, is that a knowledge of the micro-architecture of tissues, in relation to the proliferative characteristics of the subpopulations involved, is likely to be essential for a full understanding of the significance and the operation, in vivo, of cell density and cell contact factors controlling proliferation.

\section{References}

Baserga, R. (1968). Biochemistry of the cell cycle: a review. Cell and Tissue Kinet., 1, 167-191.

Blackett, N. M. (1968a). Investigation of bone-marrow stem cell proliferation in normal, anaemic and irradiated rats using methotrexate and tritiated thymidine. J. nat. Cancer Inst., 41 , 909-918.

Blackett, N. M. (1968b). Changes in proliferation rate and maturation time of erythroid precursors in response to anaemia and ionizing radiation. In Effects of Radiation on Cellular Proliferation and Differentiation; Proceedings of a Symposium, pp. 235 246. International Atomic Energy Agency, Vienna.

Block, P., Seiter, I., and Oehlert, W. (1963). Autoradiographic studies of the initial cellular response to injury. Exp. Cell Res., 30, 311-321.

Bresciani, F. (1965). In Cellular Radiation Biology: 18th Annual Symposium on Fundamental Cancer Research University of Texas, (M. D. Anderson Hospital and Tumor Institute), pp. 547-557. Williams and Wilkins, Baltimore.

Cairnie, A. B. (1967). Cell proliferation studies in the intestinal epithelium of the rat: response to continuous irradiation. Radiat. Res., 32, 240-264.

Christophers. E. (1971). Cellular architecture of the stratum corneum. J. invest. Derm., 56, 165-169.
Epifanova, O. I. (1966). Mitotic cycles in estrogen-treated mice: a radioautographic study. Exp. Cell Res., 42, 562-577.

Fry, R. J. M., Lesher, S., Kisieleski, W. E., and Sacher, G. (1963). Cell proliferation in the small intestine. In Cell Proliferation: A Guiness Symposium, edited by L. F. Lamerton and R. J. M. Fry, pp. 213-235. Blackwell Scientific Publications, Oxford.

Galjaard, H., Meer-Fieggen, W. van der, and Giesen, J. (1972) Feedback control by functional villus cells on cell proliferation and maturation in intestinal epithelium. Exp. Cell Res., 73, 197-207.

Gidali, J., and Lajtha, L. G. (1972). Regulation of haemopoietic stem cell turnover in partially irradiated mice. Cell Tissue Kinet., 5 , 147-157.

Hanna, I. R. A., Tarbutt, R. G., and Lamerton, L. F. (1969). Shortening of cell-cycle time of erythroid precursors in response to anaemia. Brit. J. Haemat., 16, 381-387.

Hegazy, M. A. H., and Fowler, J. F. (1973). Cell population kinetics of plucked and unplucked mouse skin. I. Unirradiated skin. Cell Tissue Kinet., 6, 17-33.

Hermens, A. F., and Barendsen, G. W. (1969). Changes of cell proliferation characteristics in a rat rhabdomyosarcoma before and after X-irradiation. Europ. J. Cancer., 5, 173-189.

Iversen, O. H., Bjerknes, R., and Devik, F. (1968). Kinetics of cell renewal, cell migration and cell loss in the hairless mouse dorsal epidermis. Cell Tissue Kinet., 1, 351-367.

Kember, N. F. (1960). Cell division in endochondral ossification. $J$. Bone Jt Surg., 42B, 824-839.

Lahiri, S. K., and van Putten, L. M. (1972). Location of the $\mathbf{G}_{0}$-phase in the cell cycle of the mouse haemopoietic spleen colony forming cells. Cell Tissue Kinet., 5, 365-369.

Laurence, E. B., Randers-Hansen, E., Christophers, E., and Rytomaa, T. (1972). Systemic factors influencing epidermal mitosis. Rev. europ. Etud. biol. Res., 17, 133-139.

Leblond, C. P., Clermont, Y., and Nadler, N. J. (1967). The pattern of stem cell renewal in three epithelia (esophagus, intestine, and testis). Cand. Canc. Res. Conf., 7, 3-30.

Mackenzie, I. C. (1970). Relationship between mitosis and the ordered structure of the stratum corneum in mouse epidermis. Nature (Lond.), 226, 653-655.

Marques-Pereira, J. P., and Leblond, C. P. (1965). Mitosis and differentiation in the stratified squamous epithelium of the rat esophagus. Amer. J. Anat., 117, 73-87.

Pederson, T., and Gelfant, S. (1970). $\mathrm{G}_{2}$-population cells in mouse kidney and duodenum and their behavior during the cell division cycle. Exp. Cell Res., 59, 32-36.

Pritchard, J. J. (1963). Bone healing. Sci. Basis Med. Ann. Rev., 1963 286-301.

Steel, G. G. (1972). The cell cycle in tumours; an examination of data gained by the technique of labelled mitoses. Cell Tissue Kinet., 5, 87-100.

Tarbutt, R. G. (1969). Cell population kinetics of the erythroid system in the rat. The response to protracted anaemia and to continuous $\gamma$-irradiation. Br. J. Haemat., 16, 9-24.

Threlfall, G. (1968). Cell proliferation in the rat kidney induced by folic acid. Cell Tissue Kinet., 1, 383-392.

Tubiana, M. (1971). The kinetics of tumour cell proliferation and radiotherapy. Brit. J. Radiol., 44, 325-347.

Wright, N. A., Morley, A. R., and Appleton, D. (1972). The effect of testosterone on cell proliferation and differentiation in the small bowel. $J$. Endocr., 52, 161-175.

Zajicek, G., Michaeli, Y., and Weinreb, M. M. (1972). Kinetics of the inner enamel epithelium in the adult rat incisor during accelerated eruption. Cell Tissue Kinet., 5, 35-39. 